\title{
Galeno y Freud. \\ Sobre un obstáculo para la evolución científica del psicoanálisis.
}

\author{
Raúl Fernández Vilanova ${ }^{1}$ \\ Catamarca, R. Argentina
}

La obra de Sigmund Freud ha tenido un lugar icónico en buena parte del mundo occidental durante todo el siglo XX. Pero así como en sus inicios fue un importante revulsivo del pensamiento, con el correr de los años ha perdido esa cualidad. Ocurre que tanto Freud como sus continuadores se han esforzado por mantener intactas todas las partes de su edificio conceptual y de sus categorías clínicas. Eso ha hecho que en muchas de las asociaciones integrantes de la International Psychoanalytical Association creada por él, sus ideas se transmitan a los estudiantes como un dogma obligatorio. Más como un credo, que como un conjunto de hipótesis provisionales. De este modo, con su acatamiento literal, la obra que en su origen trajo un soplo de aire fresco a la psicología y la psiquiatría de la época, se ha convertido en un obstáculo para el crecimiento científico del psicoanálisis.

Palabras clave: Galeno, Vesalio, Freud, Ferenczi, psicoanálisis, abuso sexual infantil, trauma, fantasía, teoría pulsional, transferencia, posesividad, impulso posesivo, sumisión, ciencia, doctrina, dogma.

Sigmund Freud's work has played an iconic role in much of the western world throughout the twentieth century. But just as in its beginnings it was an important catalyst for the development of thought, it has undoubtedly lost that quality over the years. It happens that both, Freud and his supporters, have struggled to keep intact every single part of his conceptual building and its clinical categories. This has led to the fact that, in many of the member associations that make up the International Psychoanalytical Association he created, his ideas are transmitted to students as a compulsory dogma, more as a creed than as a set of provisory hypotheses. Thus, with its literal compliance, the work that originally brought a breath of fresh air to the psychology and psychiatry of the time, has now become the main stumbling block for the scientific growth of psychoanalysis.

Key Words: Galeno, Vesalio, Freud, Ferenczi, psychoanalysis, child sexual abuse, trauma, phantasy, instinct theory, transference, possessiveness, possessive impulse, submission, science, doctrine, dogma.

English Title: Galeno and Freud. About an obstacle to the scientific evolution of psychoanalysis.

\section{Cita bibliográfica / Reference citation:}

Fernández Vilanova, R. (2020). Galeno y Freud. Sobre un obstáculo para la evolución científica del psicoanálisis. Clínica e Investigación Relacional, 14 (1): 123-132. [ISSN 1988-2939] [Recuperado de www.ceir.info ] DOI: 10.21110/19882939.2020.140108

\footnotetext{
${ }^{1}$ Raúl Fernández Vilanova. Psicólogo. Raúl Fernández Vilanova fue presidente de la Asociación Psicoanalítica de Madrid (APM), componente de la International Psychoanalytical Association (IPA), y del Instituto Europeo de Psicoterapia (IEP). Dirección: Calle Rio Salado s/n semi esquina con calle Rio Abaucan 4700 - Catamarca, República Argentina; Teléfono en España: +34 654181 958; Teléfono en Argentina: +54 93834354423 ; c.e. fernandezvilanova@gmail.com; Skype: fernandezvilanovaı
} 
Una teoría puede ser consistente, muy bonita, pero no reproducir la realidad,

Antonino Marciano ${ }^{1}$

Corría el siglo II de nuestra era cuando el médico, cirujano y anatomista Galeno de Pérgamo emprendió la titánica tarea de describir el cuerpo humano. Se acercaba a su fin el siglo XIX cuando el médico Sigmund Freud reorientó su formación de neurólogo hacia la comprensión de la mente humana. Aunque ambos hombres son justamente considerados pioneros en la investigación de sus respectivos campos del saber, es menos sencillo entender por qué sus enseñanzas se han extendido en el tiempo libres de toda crítica pública por parte de sus discípulos y continuadores.

En el caso de Galeno, baste señalar que la enseñanza de la anatomía humana en las universidades europeas estuvo basada en sus libros por ¡más de 1.000 años! Hasta que el médico y anatomista Andrés Vesalio (1514-1564), de origen alemán aunque nacido en Bruselas y formado como médico en París, rompe con la autoridad de Galeno en el terreno anatómico al publicar De humani corporis fabrica (Sobre la estructura del cuerpo humano, 1543). En su gran obra demuestra que el cuerpo humano que describían los libros del maestro -quien en parte se había apoyado en la disección de monos- no coincidía con lo que mostraba la investigación de esos días. La obra desencadenó la ira de los "galenistas", que le dirigieron durísimos insultos, lo acusaron de loco y trataron de explicar la falta de coincidencia señalada por Vesalio como resultado de los cambios experimentados por el cuerpo humano en esos siglos. Pero sus descalificaciones no consiguieron impedir que la comunidad médica mandara los libros de Galeno a la sección de historia de la medicina, ni que Vesalio sea hoy considerado el fundador de la anatomía moderna.

En el caso de Freud, su obra todavía se imparte literalmente como enseñanza obligatoria en una parte de los Institutos de formación de psicoanalistas de las Sociedades que integran la International Psychoanalytical Association desde hace ¡más de 100 años! Sin duda que Freud no explicó la mente humana por analogía con seres de otras especies, como había hecho Galeno. Por el contrario, apoyó sus consideraciones e hipótesis en tres fuentes distintas, muy humanas:

--Su observación clínica de pacientes y relatos de terceros.

--Su lectura de obras filosóficas, literarias, teatrales, pictóricas, etnológicas, etc. 
--Su autoanálisis, como él mismo ha llamado al análisis de sus sueños, fantasías y acontecimientos de su vida, que realizó en su relación y correspondencia con su amigo Fliess.

Pero lo que Freud produce a partir de estas fuentes, dista mucho de ser suficiente para considerar que el campo de conocimiento abierto por él se ha constituido como ciencia. Sobre todo si se tiene en cuenta que ya durante su vida se propusieron otras formas de interpretar aquellos hechos clínicos en los que él había basado sus afirmaciones.

A fines del siglo XIX, el relato de algunos de sus pacientes y de personas ajenas a su consulta había llevado a Freud a la conclusión que el abuso sexual sufrido en la infancia por parte de padres $u$ otros adultos próximos, estaba en el origen de la patología de sus pacientes. Pero poco después él se rectifica. Concluye que había sido engañado por aquellos pacientes, y que el supuesto abuso sólo había ocurrido en su fantasía. Ni siquiera dice que el abuso sólo era verdad en algunos casos. Hace una generalización, y desemboca en la idea que ser seducido sexualmente por un adulto es una proto fantasía, o sea, una fantasía universal de los niños.

Pero Freud no tuvo que esperar tanto tiempo como Galeno a que surgieran sus Vesalios. Estaba él todavía en plena producción teórica cuando fue tácitamente cuestionado por el húngaro Sandor Ferenczi, colega y discípulo suyo. En un trabajo basado en su experiencia clínica ${ }^{2}$, Ferenczi corrigió al maestro en dos aspectos fundamentales de sus teorías, mediante constataciones de extraordinario valor:

--Una parte importante de los pacientes que él veía en su consulta eran personas que en su infancia habían sido víctimas de abusos sexuales por parte de preceptores, padres u otros familiares. Esos abusos eran la causa de traumatismos que derivaban en graves patologías.

--La actitud de reserva y abstinencia afectiva del analista, preconizada por Freud para proteger su pretendida objetividad científica, era retraumatizadora. Esto quiere decir que era vivida por el paciente como la repetición de la frialdad y distancia emocional sufrida en su infancia por parte de padres y cuidadores (precisamente aquellos que debieron protegerle, evitándole el traumatismo), quienes además se mostraban incrédulos ante el relato del abuso.

El trabajo de Ferenczi estaba destinado al congreso de Wiesbaden, y recibió una expresa censura por parte de Freud. De ese modo el maestro ejerció su poder de veto sobre el más brillante de sus discípulos, quién hubiera conducido el psicoanálisis por un camino menos fantasioso. Porque conscientemente o no, Freud hizo primar una fantasía suya por sobre las evidencias que le ofrecían Ferenczi y sus propios pacientes. 
Sin duda que la fantasía freudiana sobre presuntas fantasías originarias previas a toda experiencia (seducción del niño por un adulto, representación del coito entre los padres y castración como castigo por el deseo incestuoso), configuraba un escenario mucho más florido y rocambolesco para explicar la patología psíquica que el simple (aunque terrible) abuso sexual traumático. De hecho, a aquellas fantasías de Freud le siguieron otras, vinculadas a las primeras: niños y niñas experimentarían deseos incestuosos por uno de sus padres y odio hacia el otro, al que se representarían en la fantasía como un rival cuya muerte desean, o sea el Complejo de Edipo. Tales deseos incestuosos y parricidas tendrían como consecuencia y complemento la angustia de castración y su equivalente femenino, la envidia del pene, o sea el Complejo de castración. 3

Pero la fantasía de Freud fue más lejos. En $1915^{4}$ describe las fuerzas que según él se mueven en nuestro interior y nos empujan en distintas direcciones y con distintos fines. Encuentra que una de esas fuerzas es la que nos impulsa a la conservación de la propia vida, a la que llama pulsión del Yo, en tanto que designa pulsión sexual a la que empuja a la reproducción de la vida. En el escrito mencionado Freud realiza una minuciosa descripción de la "pulsión" sexual, e imagina la existencia de una energía a la que llama libido, distinguiendo en ella una fuente orgánica, un objeto, una finalidad y una intensidad. Unos años más tarde, en 19205, reúne esas dos fuerzas en una, a la que llama pulsión de vida, y propone una segunda, siempre enigmática y opuesta a la anterior, a la que llama pulsión de muerte. Yo mismo he dado por buena esa explicación durante muchos años, todos los que tardé en descubrir que esta hipótesis freudiana, la joya del museo para los teóricos ortodoxos, es rebuscada e innecesaria para los clínicos.

Sin duda que las teorías pulsionales de Freud han sido uno de los puntos obscuros de su teoría, y uno de los que más críticas ha despertado. Es posible que la generalización de la idea de que -según él- el motor de nuestra vida es de origen sexual, despertara la resistencia de la sociedad victoriana de su época. Pero más allá de ese reparo ideológico, es cierto que se trata de una teorización abstracta e innecesariamente complicada. Con el inconveniente añadido de que los psicoanalistas obedientes de la ortodoxia freudiana la convirtieron en una de sus banderas imprescindibles, y han difundido la obscuridad del tema pulsional sobre todo el psicoanálisis.

En más ocasiones he mostrado mis diferencias con estas y otras propuestas de Freud -surgidas muchas de ellas de su imaginación y no de la práctica clínica- aunque el principal de mis desacuerdos tiene que ver con su teoría de las pulsiones, que considero completamente prescindible. Por eso, en un trabajo publicado en el número 82/2018 de la Revista de Psicoanálisis de la Asociación Psicoanalítica de Madrid (Dime que soy Tuya. Intimidad y pulsión posesiva) he defendido la idea que el motor de nuestra vida y nuestro deseo es la posesividad, 
una fuerza tan omnipresente que no se nota, que nos acompaña desde el origen de la vida bajo formas activa o pasiva, y está en la base de todos nuestros sentimientos, deseos y pasiones. Me explayo sobre el tema de modo sencillo y coloquial en el libro próximo a editarse que llevará por título Dime que soy tuya. Psicología del impulso posesivo. Una mirada sin prejuicios a la intimidad de las parejas.

Las ideas que allí expongo proceden tanto de mi practica psicoterapéutica y psicoanalítica como de mi condición humana, en el trabajo con fantasías, sentimientos y deseos y su importancia en los vínculos que establecemos. Sin embargo, para gran parte de quienes comparten esas mismas fuentes, el papel de la posesividad que está en la base de sentimientos, deseos y fantasías ha pasado desapercibido, o ha sido considerado negativo. De este modo ha quedado en la penumbra el hecho que todo tipo de relación entre las personas, interesada o generosa, planificada o espontánea, lúdica o de trabajo es alguna de las formas en que nos poseemos los unos a los otros, como bien lo ilustran los fenómenos de transferencia que existen no sólo en la consulta terapéutica. Cada una de tales relaciones se establece mediante negociaciones y contratos muchas veces tan sutiles que se hacen imperceptibles, en los que cada uno, si compra, paga entregando algo suyo, o entregándose al otro. Con ello establecen algún tipo de posesión recíproca, de la que con frecuencia no son conscientes. Esto es aún más evidente en las relaciones amorosas y sexuales en las que los amantes se poseen en un vínculo físico y emocional, en el cual es posible reconocer las diferencias entre la posesividad masculina y femenina, así como sus distintas intensidades. Si se está advertido, es fácil de reconocer el punto en el que la imprescindible posesividad recíproca que enriquece y preserva los vínculos va siendo sustituida por distintas formas del desamor, incluido el desprecio, el odio y la violencia, mediante los que nos retiramos del juego posesivo recíproco.

Aunque no es posible hacerlo aquí, tendría interés explorar el modo en que los sujetos nos situamos respecto de nuestra posesividad en los diferentes desarrollos psicopatológicos. Simplificando mucho, los trastornos más clásicos se podrían distribuir entre aquellos en los que la posesividad falta, se hace excesiva o se desvía de su supuesto objeto natural.

Es fácil ver que el disfrute de la posesividad activa y pasiva es lo que resulta impedido en las clásicas inhibiciones de la sexualidad, que han sido asociadas a la histeria: la impotencia en el hombre y la frigidez en la mujer. Ambos padecimientos tienen en común la desaparición del deseo físico, o sea, la inhibición del impulso posesivo, que puede ser general o selectiva.

El propio Freud trató de explicar la etiología de ciertos trastornos del "Hombre de los lobos" relacionándolos con un 'coito a tergo' supuestamente observado a sus padres en la infancia, 
que le habría despertado gran excitación e intensos deseos activos y pasivos, de dominación y sometimiento.

En un sentido opuesto a la inhibición, la posesividad activa y pasiva está exacerbada en sádicos y masoquistas. La pareja sadomasoquista disfruta de la intensidad incrementada de los sentimientos de entrega y posesión. El dolor inferido, o el dolor sufrido y aceptado, no es atractivo en si mismo para los actores, sino un signo valioso de la posición activa o pasiva que disfrutan. Ni que decirse tiene que para la mayoría de las personas son inconfesables los placeres posesivos, ni siquiera los de baja intensidad comparados con aquellos. Aunque no sea posible representarnos la relación de dos personas que se desean sin contar con la referencia a la posesión y la entrega.

No hay aquí espacio para argumentarlo, pero cabe pensar que la posesividad, como escenario común de las relaciones, desaparece en las psicosis, o toma formas bizarras: delirios esquizoparanoides de ser influido o poseído, obsesión esquizofrénica por dominar el origen y el sentido -que a él se le hace evanescente- de las palabras y las cosas.

Se que toco creencias profundamente arraigadas en buena parte de la comunidad psicoanalítica, pero ¿por qué pensar que necesitamos una palabra específica, la tan popular como enigmática libido, para referirnos al empuje sexual hacia el objeto? Sin duda que ese empuje sexual existe, y más aún, es el paradigma de la posesividad. Sin duda que ese empuje nos hace investir el objeto, que no es otra cosa que convertirlo en algo a poseer de alguna de las maneras posibles. Es tan universal nuestra naturaleza posesiva, es tan omnipresente la posesividad, que es el tejido conjuntivo de toda nuestra vida psíquica y social. Naturalmente, cuando hablo de poseer no me refiero sólo a lo que pasa en todo el repertorio del deseo y la atracción sexual, donde es evidente. Es posesiva también la relación con nuestros hijos, padres y amigos. No se trata de dominio, sino de un poderoso sentimiento subjetivo vivido por cada uno de nosotros con distintos gradientes respecto de todo lo que nos importa.

Hecha esta digresión que cuestiona la teoría pulsional freudiana, entiendo que es muy posible que la marcha y contramarcha de Freud respecto del abuso infantil traumático tenga que ver con que esa teoría aparecía como demasiado simple para su ambición intelectual. Sin duda que al suprimirse la realidad del abuso y poner en su lugar la fantasía, se abre un gran horizonte a la teorización. Pero, así como en el mundo de los medios de comunicación se ironiza sobre ciertos periodistas diciendo que no dejan que los hechos les estropeen un buen titular, podríamos decir que Freud rechaza la realidad del traumatismo para que no haga sombra a su alambicada teoría pulsional.

La tenacidad con que Freud fue desarrollando, arborizando y difundiendo sus teorías le rindió provecho. Pronto contó con un grupo de médicos, psicólogos e intelectuales de la época ante 
quienes sus ideas eran un soplo de aire fresco. Pero de ser un novedoso revulsivo a atribuirle un estatuto científico per se, había una gran distancia que no se debió ignorar. Si se hizo fue en parte porque Freud ofrecía una original y estructurada visión del psiquismo, y en parte por el fenómeno de la transferencia, muy bien estudiado por él y aplicado en su trabajo Psicología de las masas y análisis del Yo. Allí se ve que la capacidad de influencia del líder carismático se nutre de las humanas necesidades de sometimiento y pertenencia a un grupo. Eso hizo que las hipótesis freudianas, que deberían haber pasado por un cuidadoso proceso de selección ${ }^{6}$ y verificación, fueran adquiriendo para sus seguidores el carácter de un saber revelado y oracular, imposible de ser sometido a la duda o la falsación popperiana. Pero más que eso. EI edificio conceptual freudiano ha permanecido inalterable a lo largo de los años, inmune a los desarrollos de otros autores incompatibles con él, hasta el punto de convertirse en la religión común de una amplia parte de los psicoanalistas. Y así las cosas, son pocos los que levantan el dedo diciendo que con el correr del tiempo el rey ha quedado desnudo.

Me he visto empujado a pensar en la pretensión de cientificidad de un psicoanálisis que reivindica la vigencia de las teorías freudianas en bloque, en dos circunstancias diferentes. Una de ellas, compartida con la mayoría de mis colegas, ha ocupado gran parte de mi vida. Es la de alguien preocupado por conocer el funcionamiento de la mente y aliviar los padecimientos psíquicos. Ambos intereses son los que me han llevado a hacerme psicoanalista y se mantienen vivos en la actualidad. La otra circunstancia es más recortada en el tiempo. Se extiende desde diciembre de 2009, cuando me eligen Secretario Científico de la Asociación Psicoanalítica de Madrid (APM), componente de la International Psychoanalytical Association, hasta noviembre de 2016, cuando renuncio al cargo de presidente de la APM, que desempeñé durante tres años.

Seguramente las responsabilidades de gestión asumidas durante aquellos siete años me pusieron por delante cosas curiosas que ya no pude dejar de ver. Una de ellas fue que como Secretario Científico me tocaba organizar actividades "científicas", y pude sentir que algo se revolvía dentro de mí al tener que anunciarlas de esa manera. Fue entonces que comencé a sentir más fuertemente que el adjetivo "científico", aplicado al aparato conceptual producido por S. Freud, era una sobrecompensación de sus carencias epistemológicas, y teñía de impostura una práctica que de otro modo debiera ser considerada digna, honesta y útil. Una práctica rica en intuiciones sobre el que probablemente es el territorio más difícil de la ciencia, pero que necesita soltar de una vez amarras con la obra de Freud, enviándola a los estantes de Historia del psicoanálisis, para llegar a exhibir credenciales científicas algún día.

La otra cosa "curiosa" a la que me refiero se me acabó de hacer evidente durante mi presidencia de la APM: el tipo de formación que impartíamos a nuestros candidatos no era 
científica sino doctrinaria. Para aclarar qué quiero decir con esto, basta indicar que los únicos seminarios obligatorios que dábamos en nuestro "Instituto de Formación" tenían como tema la obra de Sigmund Freud, repartida en cuatro períodos cronológicos. O sea que una obra cuya elaboración concluye con su muerte en 1939, se impartía en 2016 como una suma de axiomas "científicos" que ni necesitan ni admiten discusión. O sí, pero una discusión complaciente, de la que cada palabra de Freud salía indemne, aunque se diera de patadas con otras palabras suyas o de otros autores. O sea que no se tomaba esa obra como un conjunto de hipótesis originales (aunque también plagada de incoherencias, cambios de rumbo y afirmaciones sin otro apoyo que la autoridad del autor) que buscan una aproximación a la verdad, sino como un dogma de fe que se coge o se deja en su conjunto. Cada uno de los recodos del camino recorrido por Freud durante casi 50 años -originados muchos de ellos en su frondosa imaginación-, aparecía ante nuestros alumnos tan importante como todos los demás recodos. Sin duda que el "barrido epistemológico" descrito por Khun como el recurso fáctico con que las ciencias depuran los conceptos superados, estaba completamente desactivado en nuestra práctica docente.

Ante el acatamiento religioso a Freud por parte de sus maestros, que convertía la enseñanza en adoctrinamiento, ¿cual era la actitud de los alumnos? La sumisión. O una crítica sólo aparente, que tenía un tope muy claro, establecido por el propio Freud, y que todavía hoy constituye el santo y seña de gran parte de los psicoanalistas de IPA: quién pretenda ser considerado psicoanalista debe asumir la teoría de las neurosis (en la que el abuso precoz real no existe) y la de una sexualidad infantil moldeada por los complejos de Edipo y Castración, así como las teorías del inconsciente, represión, pulsión y transferencia. Son nociones de muy distinta importancia y significado, porque así como la transferencia es un hecho incontestable que todo terapeuta puede observar en la clínica con independencia de su orientación, no cabe decir lo mismo del resto de esas nociones. Sin embargo, aún hoy respetables colegas se ven en figurillas para expresar libremente su pensamiento sin correr el riesgo de chocar con alguno de los intocables conceptos de Freud.

Y los psicoanalistas formados en esa ortodoxia ¿qué hacen en su práctica clínica y docente? En eso hay diferencias. Están por una parte aquellos que entienden la enseñanza del psicoanálisis como un lecho de Procusto para ahormar psicoanalistas "freudianos". Identificados con la doctrina, han acuñado la expresión "identidad psicoanalítica" para describir su convicción radical. Un nombre con resonancias religiosas que envuelve, unifica y proporciona un confortable sentido de pertenencia a quienes no tienen ninguna duda sobre la verdad "científica" de cada una de las ideas y palabras del maestro. Han renunciado a pensar por su cuenta y como discípulos fieles asestan esas "verdades" a alumnos y pacientes. 
Hay otros que poco a poco han llegado a notar que aquellas ideas teóricas y categorías clínicas, como le pasó a Vesalio con Galeno, no reflejan lo que ellos ven en su práctica. Y al darse cuenta pensaron primero que eran ellos los equivocados, que se trataba de un defecto suyo. Sólo poco a poco se atrevieron a pensar que, si las cosas que seguían viendo en la clínica no coincidían con la descripción de Freud, podían estar indicando que el equivocado era Freud. Y aquí nuevamente hay dos grupos.

Una parte de los que recuperaron su pensamiento y se sintieron con derecho a usarlo, tomaron distancia de la doctrina oficial, y en la medida de su resolución y sus posibilidades trataron de avisarle al resto (iiOiga, mire, que esto no es lo que parece!!). Desecharon el camino oficial pavimentado y bien señalizado y aceptaron la incomodidad de ir campo a través. Trataron de evitar cuestionamientos directos al maestro, pero no se privaron de ofrecer explicaciones alternativas a buena parte de los problemas abordados por él. Es el caso de gran cantidad de autores que vienen haciendo aportaciones decisivas al desarrollo del psicoanálisis desde antes y después de la muerte de Freud.

Otros, más pragmáticos, quizá sintiendo que con la iglesia habían topado, sabiendo que el pensamiento no tiene tanta fuerza como la fe, se resignaron a discrepar de Freud sólo en la intimidad. Sobrellevaron en silencio la distancia entre un sistema de ideas en que ya no creían y una práctica clínica honesta y eficaz, pero sólo basada en intuiciones propias, que lamentablemente no podían beneficiarse del enriquecimiento de la discusión abierta por la tiranía del dogma.

Son dos posiciones respetables. Una parte de mí me decía "no vale la pena el esfuerzo de levantar la voz". Esa parte prefería la segunda opción. Pero ahora veo que se me impuso la primera, a partir de darme cuenta de que la obra de Freud y sobre todo su metapsicología, los ilusionantes revulsivos de otra época, se había convertido en un dogma que impedía la consolidación científica del psicoanálisis. Como es obvio, no será la minuciosa lectura y relectura de Freud lo que nos permita ingresar con seriedad al obligado territorio de la ciencia ni la clínica actual. Por la especial naturaleza de su objeto, que es tanto biológica como simbólica, el psicoanálisis está llamado a entenderse con las modernas neurociencias, que tienen cosas que decir -y están diciendo-sobre energías, impulsos y la base neurológica los procesos psíquicos. Y también, puesto que trabaja con signos, símbolos y representaciones, con la semiótica de orientación peirceana, esencial como teoría del insight y de la organización de la mente?.

Original recibido con fecha: 1/12/2019 Revisado: 11/2/2020 Aceptado: 15/03/2020 expresa. Este material es para uso científico y profesional exclusivamente y puede contener información clínica sensible. Los editores no se responsabilizan de los contenidos de los autores. Dirigir las consultas sobre derechos y autorizaciones a ceir@psicoterapiarelacional.es 
NOTAS:

${ }^{1}$ (Roma, 1979). Físico teórico que investiga en Shanghái desde el año 2013.

2 Sandor Ferenczi, La confusión de lenguajes entre el adulto y el niño, 1932.

${ }^{3}$ Reservo para otro trabajo una revisión crítica de las principales ideas de S. Freud.

4 Sigmund Freud, Las pulsiones y sus destinos, 1915

${ }_{5}^{5}$ Sigmund Freud, Más allá del principio de placer, 1920

${ }^{6}$ No podemos olvidar que en muchos lugares de su obra hay más de una respuesta a un mismo problema conceptual.

7 --Aportes de la Neurociencia y la Semiótica a la teoría y clínica de las patologías tempranas. Alejandro Guiter Viader, Revista de Psicoanálisis (Madrid) 2016; № 77

--Teoría y clínica de la Escisión del Yo. Raúl Fernández Vilanova y Pedro Gil Corbacho, Revista de Psicoanálisis (Madrid) $2012 ;$ № 64

--Psicoanálisis del efecto mariposa. Alguna idea sobre el origen, funcionamiento y abordaje de la escisión y la regresión traumáticas. Raúl Fernández Vilanova, 1998; Trabajo de acceso a Miembro Titular de la APM. Inédito 